\title{
Calibration of an eye-movement system for use in reading
}

\author{
CÉCILE BEAUVILLAIN \\ Université René Descartes, Paris, France \\ and \\ PIERRE BEAUVILLAIN \\ Université Paris-Sud, Orsay, France
}

\begin{abstract}
A method for measuring horizontal eye movements in the msec range is described. Accurate measurement of horizontal eye movement over a linear range of $12^{\circ}$ is achieved by processing the image of the eye illuminated with infrared light and with the head position fixed. The system has given very reliable results, and a resolution of $6 \mathrm{~min}$ of visual angle can be achieved with a character space of $45 \mathrm{~min}$ of arc. We also describe efficient numerical-data processing which allows the precise determination of the absolute position of the eye.
\end{abstract}

Accurate recording of eye movements is a prerequisite for behavioral investigations of reading. A comprehensive summary of methods for recording eye movements was given by Young and Sheena (1975). There has been considerable development in eye-monitoring technology, and some of the present systems appear to be easy enough to use, while at the same time meeting the requirement of accurate recording. The degree of data accuracy that is needed for investigating reading varies as a function of the questions asked in the research. Some reading studies only need precise data on the duration of fixations, while others need an accurate indication of the beginning of a saccadic movement in order to change the display, and still others need an accurate indication of exactly where in the word the eye was directed on a given fixation. For example, the degree of accuracy required for investigating the effects of parafoveal information on the landing position in a word is high-indeed, the observed effects are generally about one character space of saccade in amplitude when different types of parafoveal information are tested (see, e.g., Morris, Rayner, \& Pollatsek, 1990). This type of study thus requires great precision and information concerning the accuracy of the data. The purpose of this paper is to report information we used concerning numerical-data processing in order to determine precisely the absolute position of the eye.

In our system, we used an infrared oculometer commercialized by Bouis (Bach, Bouis, \& Fischer, 1983). In

We would like to thank Raphaël Boggio-Pasqua and Michel Cochin of the Ecole Nationale d'Electronique et de ses Applications, Cergy, France, for technical assistance in programming eye-movement processing. We also thank Pierre Dana of the Institut Dentaire of Paris for very helpful suggestions concerning the submaxillar dental print technique. Correspondence concerning this article should be addressed to C. Beauvillain, Laboratoire de Psychologie Expérimentale, 28 rue Serpente, Paris 75006, France (e-mail: labexp@frmop22.cnusc.fr). this apparatus, the eye is homogeneously illuminated with infrared (IR) light $(950 \mathrm{~nm})$ using an infrared mirror. The IR light is generated by 36 light-emitting diodes (LEDs). This type of LED has an integrated lens that produces a narrow-beam profile of $15^{\circ}$ full width at half magnitude. Therefore, the total radiant flux of $0.1 \mathrm{~mW}$ covers the eye diffusely, at a distance of $15 \mathrm{~cm}$. The use of a large number of LEDs, each of which operates at the low current of $12 \mathrm{~mA}$, minimizes radiation hazard to the retina, since each source emits only a small amount of energy. A lens focuses the IR image of the eye at a $2 \times$ magnification on a position-sensitive $20 * 20 \mathrm{~mm}^{2}$ photo diode. The position of the eye is measured via the center of gravity of the distribution of the light falling on the photo diode.

Figure 1 shows the intensity profile of the IR-reflection eye image. Three different light intensities can be discriminated, the highest intensity coming from the white sclera, the middle one coming from the iris, and the lowest one coming from the pupil. Basically, the position of the eye is electronically computed from the distribution of the light both horizontally and vertically. As this technique uses the complete picture of the eye instead of the picture of only one particular point, it is not sensitive to local irregularities of the cornea. Gaze direction is measured by analyzing the center of gravity of the first Purkinje reflections, as well as the entire IR image of the eye. To eliminate any disturbance caused by ambient illumination, the current of the infrared LEDs is cut off at $3 \mathrm{kHz}$, and the detection electronics use lockin amplifiers. For adjustment and calibration, the sensor is roughly aligned by mechanical means, using a nightviewing IR-image converter tube instead of the photosensor to move the image of the eye into the center of the sensor. The unit is then further adjusted mechanically to give zero-output voltage when the subject fixates straight ahead. 


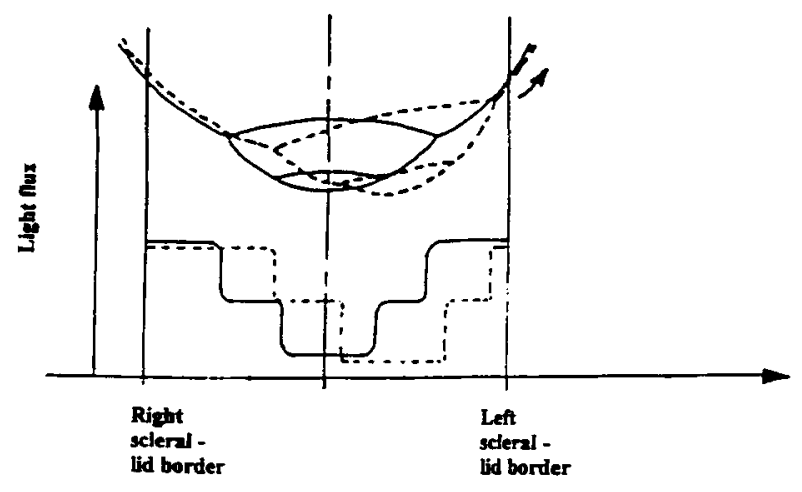

Figure 1. Schematic view of two IR-reflection eye images (solid and dotted lines) correlated to a scheme of the eye during two successive fixations. Three different light intensities, coming from the white sclera, the iris, and the pupil, are discriminated by the oculometer system. The entire IR image moves in the direction of eye movement.

The main difficulty in using this device to full advantage is that, to do so, it is necessary to prevent head movements. Because this commercial system was mainly used with monkeys performing visuo-oculomotor tasks (Fischer \& Kunz, 1981), this presented no problem, since the monkeys' heads could be tightly fixed. In order to measure the gaze direction of humans, however, it has been necessary first to overcome errors due to head movements. We have therefore built a submaxillar dental print using dental paste, allowing the submaxillar to be rested without biting. This dental equipment can be modulated for each subject in the three spatial dimensions. Added to the headrest, it ensures head stability in a relatively comfortable fashion. In our application, numerical sampling with a 12-bit analogic-digital interface occurs once ever $2 \mathrm{msec}$ during reading tasks, and once every $7.5 \mathrm{msec}$ during the calibration task. Data acquisition is performed over a horizontal angular range $\left(-6^{\circ}\right.$ to $\left.+6^{\circ}\right)$, with an output voltage of between -2 and $+2 \mathrm{~V}$ ). One bit corresponds to $5 \mathrm{mV}$, which is lower than the electronic noise of the oculometer system $( \pm 15 \mathrm{mV}$ without analogic filtering).

\section{METHOD OF CALIBRATION}

A calibration method was used whereby the output of the eye-movement monitor was recorded while a subject fixated several calibration points. Subjects were required to track the displacement of a target from one position to another $3^{\circ}$ away. The calibration target consisted of a vertical bar $\left(0.5 * 5 \mathrm{~mm}^{2}\right)$ that equaled $23 \mathrm{~min}$ of arc of vertical visual angle. Five calibration bars were successively displayed to cover the location points of a $12^{\circ}\left(-6^{\circ}\right.$ to $\left.+6^{\circ}\right)$ visual angle used in the experimental reading task. At the end of a calibration procedure, subjects again had to fixate the central calibration target. The computer sampled the voltage values corresponding to each target location. To prevent fatigue while fixating a point from being a large source of variations, each of the calibration bars was flickered at a rate of $150 \mathrm{msec}$ for a total duration of $1,200 \mathrm{msec}$.

\section{IDEAL CALIBRATION SIGNAL AND SACCADE DETECTION}

The ideal signal corresponding to the five calibration fixations is shown in Figure 2. It consists of five regular steps in time and voltage. The last central calibration target is not taken into account in the data processing. For an amplitude of $3^{\circ}$, the average saccade duration of our subjects was about $30 \mathrm{msec}$. To detect the beginning and the end of a saccade, we used the numerical derivative (Coëffé, Humbert, Jacobs, \& O'Regan, 1982). The time derivative, $d(t)$, of the signal, $s(t)$, output calibration signal is $d(t)=s(t)-s(t-D)$. As explained later, in order to detect either saccades or blinks, D was empirically chosen to be $90 \mathrm{msec}$. Strong peaks with a time shift of $D / 2$ evidenced saccades. We automatically detected them with a threshold voltage criterion. The ideal amplitude of the derivative $d(t)$ is $1 \mathrm{~V}$ for $\mathrm{a} 3^{\circ}$ saccade. The voltage threshold was chosen as $250 \mathrm{mV}$ in order to detect all main calibration saccades and blinks.
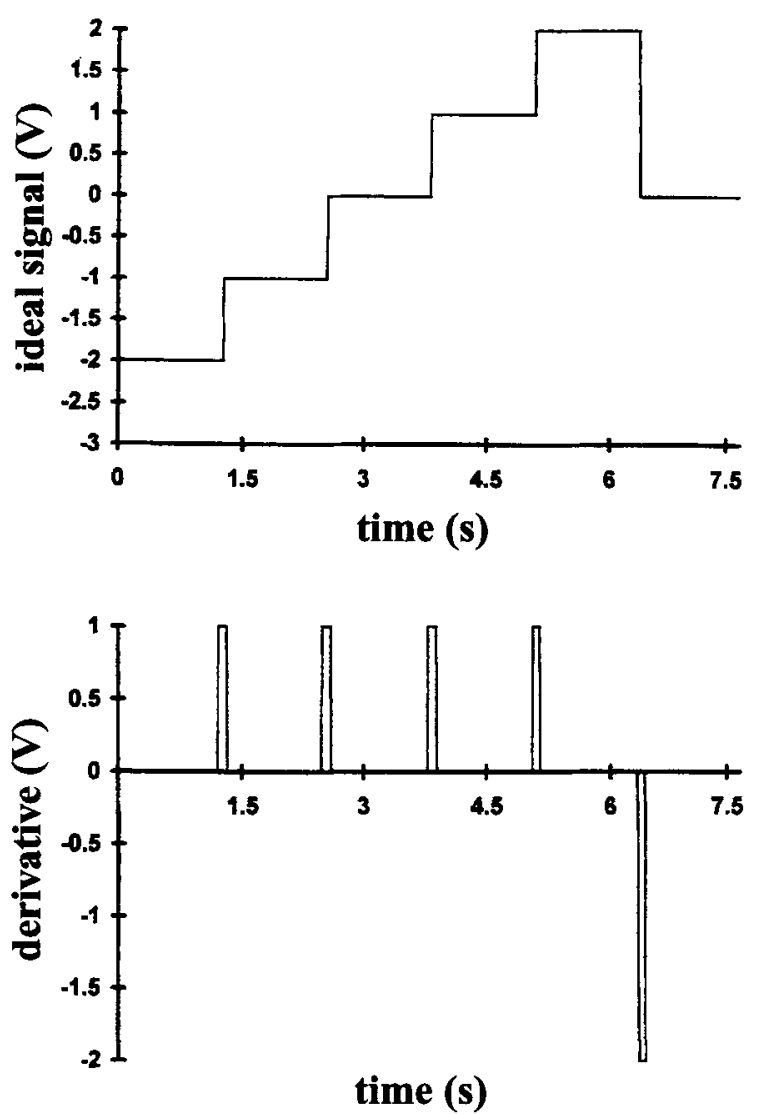

Figure 2. Ideal signal (top) and derivative (bottom) corresponding to the five successive $3^{\circ}$ horizontal eye displacements of the target during the calibration task. 


\section{REAL SIGNAL PROCESSING AND NOISE DETECTION DURING CALIBRATION PROCEDURE}

After identifying the beginning and the end of saccades, algorithms are required that process the real signal and detect noise and disturbances in the calibration data. Thus, blinks have to be detected and discarded from the data in the calibration phase. Blink signals during the calibration task can be seen in Figure 3, and Figure 4 shows the calibration-data processing of this signal.

The blink is characterized by a sign change in two derivative successive peaks (Figure $4 \mathrm{a}$ ). To discriminate between real blinks and eye saccades, the blink criterion is validated if the derivative signal has opposite signs for successive extrema. In this case, the two absolute maxima must have a ratio of between $1 / 4(250 \mathrm{mV})$ and 4 , as the speeds of the closing and opening of the eyelids may slightly differ. Because the mean saccade duration is about $30 \mathrm{msec}$ and the duration of a blink is far longer than $150 \mathrm{msec}$, we chose a $\mathrm{D}$ value of $90 \mathrm{msec}$ in the derivative calculation in order to enhance amplitude of blinks at the respective maxima. During the calibration procedure, the blink data are canceled after detection. These canceled data, filled by the mean value of the fixation step in all the figures, are not taken into account in the data processing (Figure 4b). Before the mean values of each fixation step of the calibration are determined, the derivative is computed once again without eventual blink data in order to detect small-amplitude corrective saccades (Figure 4c). Indeed, during the calibration phase, tracking saccades have to foveate the calibration spot and refoveate the spot that changes this position while being fixated. Saccades frequently do not accu-

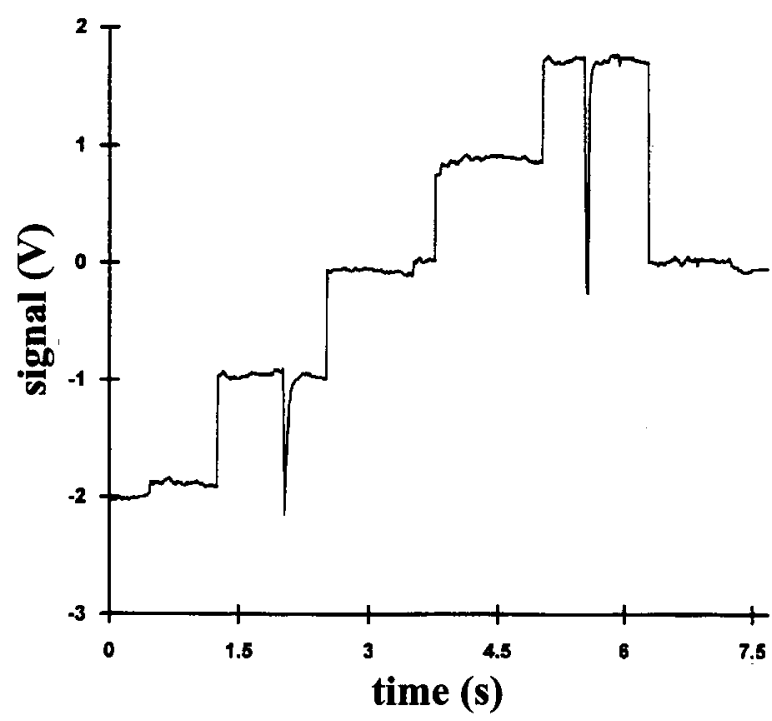

Figure 3. Example of a calibration signal over time during a calibration task over $12^{\circ}$ of visual angle. Blink signals are observed during the second and fifth fixations.
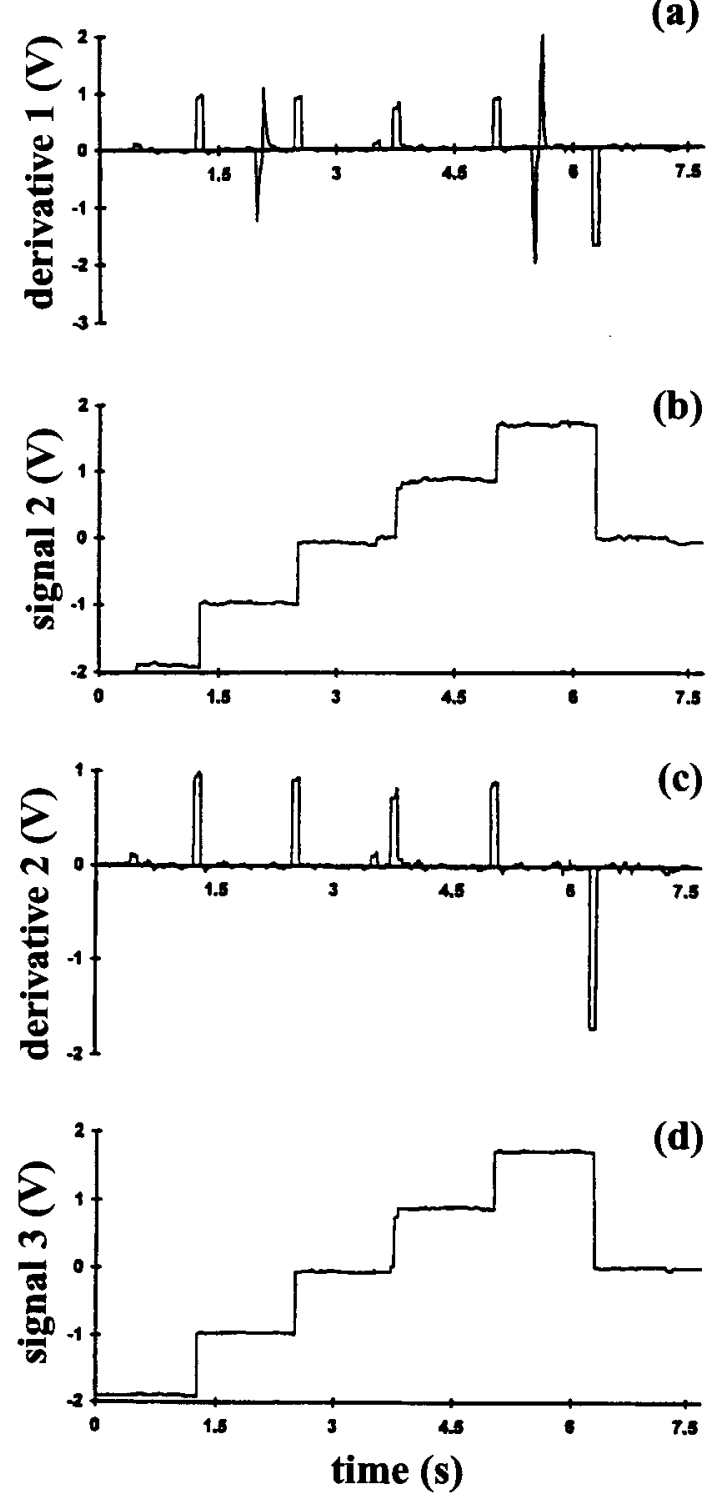

Figure 4. Processing of the calibration signal shown in Figure 3. (a) Derivative of the real signal; (b) real signal when values corresponding to blinks were canceled and replaced by the values of the eye before and after blinking; (c) derivative after blink cancelation; and (d) real signal after points more than $\pm 1 S D$ were canceled.

rately hit the target at which they are aimed. Corrective movements are then required to eliminate the step-size errors. These corrective movements follow upon the end of the main distance and saccade amplitude. The error amplitude, $\mathrm{E}$, is defined as the difference between $\mathrm{T}$, the target distance, and saccade amplitude (i.e., $\mathrm{E}=\mathrm{T}-\mathrm{A}$ ). A negative sign of $E$ indicates an overshoot of the saccade, while a positive sign indicates an undershoot. The frequency of such errors increases when subjects try to anticipate an expected target and initiate saccades before it appears, or less than $80 \mathrm{msec}$ after its appearance (see Figure $4 \mathrm{~b}$ ). When the eye position does not correspond 


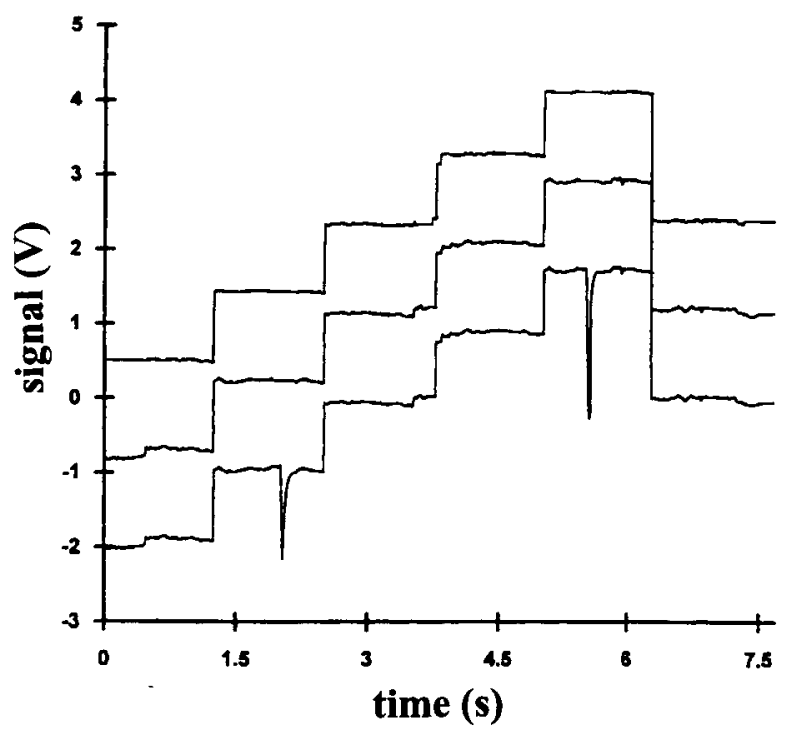

Figure 5. Successive-data processing on the calibration signal shown in Figure 3. A shift of $1.2 \mathrm{~V}$ was applied to the successive signals for a clear presentation of the data processing.

to that of the target, the eyes will remain at the incorrect position for a period of $100-150 \mathrm{msec}$ while a secondary saccade is elaborated. This kind of corrective eye movement is frequently observed during the calibration task. Indeed, such a task is artificial and differs markedly from reading. If the first period is clearly shorter than the period that follows the correction (e.g., 200 vs. $1,000 \mathrm{msec})$, the values of this corrective latency are canceled from the mean fixation (Figure $4 \mathrm{~d}$ ). If this time criterion is not followed, the calibration data are rejected and a new calibration procedure is automatically applied. A clear indication of the different steps in the data treatments described earlier is given in Figure 5.

Other corrective movements, called glissades, follow upon the end of the antecedent saccade, but with no latency. The eye moves very slowly and at a rather constant velocity. In this case, the standard deviation $(S D)$ is great and the data above $\pm 1 S D$ are discarded from the analysis. To detect these glissades, the slope of the drift is computed by least square fits on the remaining data, and calibration is considered to be wrong when the slope is too great.

Following this first signal processing in which disturbances in the signal that correspond generally to blinks and corrective saccades are discarded from the calibration data, a new calibration task is performed by the subjects when the number of saccades is greater or smaller than expected. To the extent that the number of saccades and fixations is true, the data above $\pm 1 S D$ are discarded from the mean gaze direction adopted by the eye on each calibration point (Figure $4 d$ ).

Thus, the mean gaze directions constitute what is referred to as the calibration table. This table provides a means of interpolating between these known points to assign stimulus locations to eye-movement-signal val- ues in reading. This requires testing the linearity of the calibration-table values within the $12^{\circ}$ visual angle. Indeed, in order for a linear-interpolation approach to be taken in mapping from eye signals to stimulus locations in reading, the differences between eye-signal values corresponding to each of these calibration locations have to be linearly related. To the extent that the mean values corresponding to two or more calibration targets have a linearity error that exceeds the expected linear value by $15 \%$ or more, the calibration is rejected. When it is only the mean value corresponding to one calibration target that is not linearly related to the other values (i.e., that exceeds linearity error by $15 \%$ or more), this value is not taken into account in the calibration-factor calculation. When the calibration is considered to have been successful, a calibration factor is calculated as the mean value of the five calibration steps. As the calibration targets are separated by a $3^{\circ}$ visual angle, the calibration factor gives a precise relation between the voltage output of the oculometer system and eye position (the latter given in terms of visual angle). A typical value of the calibration factor in our experimental set-up is about $900 \mathrm{mV}$ for a $3^{\circ}$ saccade for standard IR intensity.

\section{CONTROL OF THE LINEARITY OF THE OCULOMETER SYSTEM}

The use of linear interpolation requires a linear-eyemovement recording system and a precise indication of the range of visual angle over which the system is linear. To test the linearity and the ultimate resolution of the oculometer with numeral acquisition and data processing, we have processed 252 calibration data performed over $12^{\circ}$ of horizontal angle by 18 subjects. Each subject performed 14 calibration tasks. As the calibration factor slightly differs between subjects as a function of the IR reflection of the eye, the calibration data were normalized to $1 \mathrm{~V}$ for $3^{\circ}$ of visual angle. The mean deviation of the output voltage from the ideal signal corresponding to each of the five steps was calculated on these normalized data.

Figure 6 presents the mean deviation for the 252 calibration data. The deviation from linearity within $12^{\circ}$ of visual angle is negligible, and is better than $\pm 3 / 1,000$. Figure 6 clearly shows that the observed nonlinearity is mainly due to the screen curvature, with a screencurvature radius of about $1 \mathrm{~m}$. The typical fluctuation due to eye movement and the oculometer system during a calibration-target fixation is shown in Figure 7. The calculated standard deviation during a calibration-target fixation is $9 \mathrm{mV}$. The estimated error indicated in Figure 6 corresponds to $3 \mathrm{~min}$ of $\operatorname{arc}\left(9 \mathrm{mV}^{*} 1.65, p<.10\right)$.

\section{REAL SIGNAL PROCESSING AND NOISE DETECTION DURING READING}

The following data processing is designed to sample eye position when subjects read an isolated word displayed on a horizontal line. Each reading trial was per- 


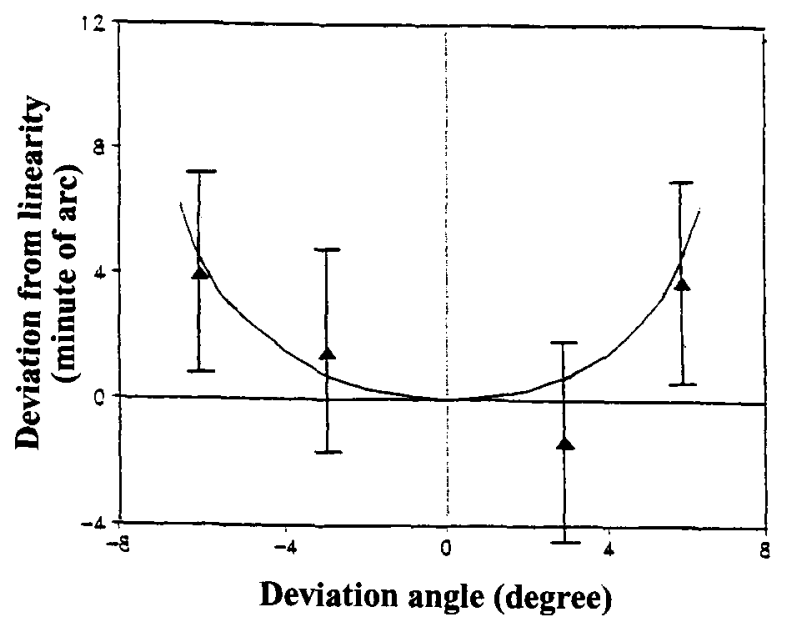

Figure 6. Deviation from linearity as a function of deviation angle. The solid line represents the calculated deviation due to the screen curvature; error bars correspond to $\pm 3 \mathrm{~min}$ of arc (estimated error for calibration fixation).

formed while subjects were required to fixate two calibration points before and after reading an isolated word. These two fixated points correspond to the starting offset $\left(-6^{\circ}\right.$ visual angle $)$ and the ending offset $\left(+6^{\circ}\right.$ visual angle). To increase the accuracy of the eye-movement detection in reading, the eye location was sampled at a rate of $2 \mathrm{msec}$, though the analogic output was filtered at $150 \mathrm{~Hz}$. The analogic filtering introduces a small delay of about $3 \mathrm{msec}$ in the detection of the onset of a saccade. To detect the beginning and the end of a saccade, we used the numerical derivative $d(t)$ of the $s(t)$ output reading signal, $d(t)=s(t)-s(t-D)$. As the saccade detection generally observed in our data is in the $30-50$-msec range for a saccade length of about 6 character spaces $\left(4^{\circ}\right)$, D was put at $20 \mathrm{msec}$ in order to also detect small eye movements.

Figure 8a illustrates an eye-movement signal observed in reading a seven-letter word, as the calibration factor was $269 \mathrm{mV}$ for a $45^{\circ}$ character space. The beginning and the end of the saccades and fixations were computed from the derivative signal in Figure 8b. In the Figure 8b, the beginning of the first saccade is clearly discriminated at $212 \mathrm{msec}$, with a total saccade duration of $54 \mathrm{msec}$. This first saccade is followed by a stable fixation at the 3.6 letter landing position, and then by a small regressive saccade of 0.2 character space at $542 \mathrm{msec}$ within the same third character space. This regressive saccade is clearly identified in the derivative of the signal, with a duration of $28 \mathrm{msec}$. After this regressive fixation, the eye makes a saccade of 34-msec duration at the 5.7 letter position. A dynamic overshoot is clearly exhibited at the termination of this third saccade, followed by a stable fixation of $196 \mathrm{msec}$. Such a dynamic overshoot occurs in a substantial proportion of saccades in reading. In this example, and as usually observed, the eye returns to its final position within $15 \mathrm{msec}$ (Kapoula, Robinson, \& Hain, 1986). Such small regression movements are correlated with the termination of a saccade and may be easily distinguished from the regression that occurred within the third letter position. Notice that the accuracy of the system allows detection of small regression movements within a character space. Obviously, these types of movements - overshoots, undershoots after a saccade, and regressive movements-are taken into account in the word-data analysis.

Figure 9 shows the noise of the eye signal during the last fixation on the seven-letter word presented in Figure 8 . It can be seen that the $S D(7 \mathrm{mV})$ during the reading task is slightly less than that observed during the fixation of a calibration target.

\section{DEGREE OF SHORT- AND LONG-TERM REPEATABILITY}

The accuracy in eye-movement data also depends on the degree of repeatability of the eye-movement signal. When engaging subjects in the reading task, it is necessary to control some changes that occur in the calibration values over the period that data are being collected. McConkie (1981) has suggested that sources of inaccuracy in eye-movement data can be due to errors that reduce short-term repeatability of the eye-movement signal. These include noise in the eye-movement system and inability of subjects to reposition their eyes accurately. Another source of errors develops during the experimental phase, and might be called long-term repeatability. It results primarily from the head movements, but also from various types of problem in the dynamic operation of the oculometer itself, as well as from lack of consistency in eye position when the subjects are asked to look at the same location.

To control this short- and long-term repeatability, the calibration table is updated during the experimental

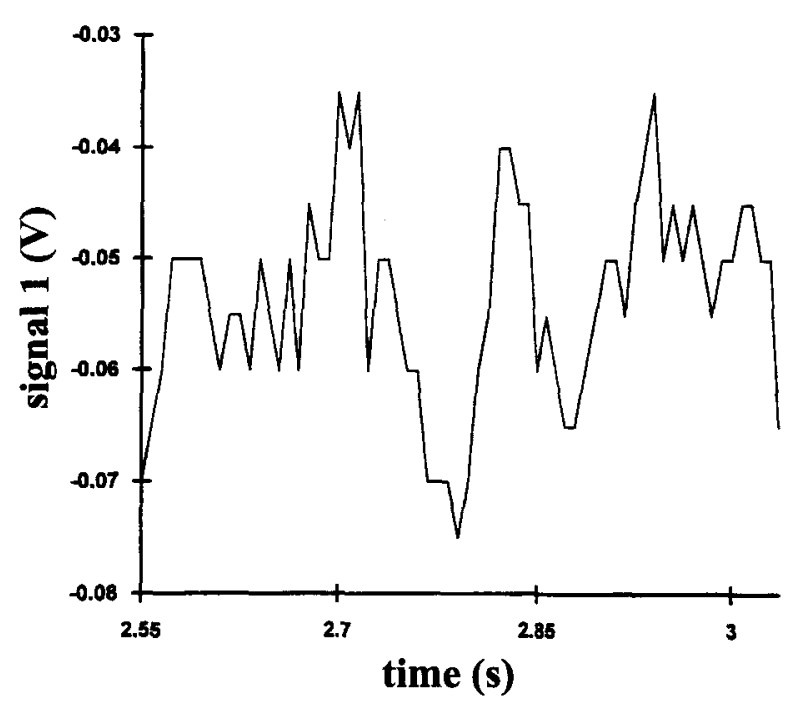

Figure 7. Noise in the eye signal over time during a calibrationtarget fixation, as observed in a part of the third calibration-target fixation shown in Figure 3. 
phase by systematically performing on each experimental trial two calibration controls, one on the calibration point, corresponding to a visual angle of $-6^{\circ}$ (the starting offset), and a second one corresponding to a visual angle of $+6^{\circ}$ (the ending offset). The calibration values obtained prior to and following the reading of a horizontal line are compared with that obtained in the calibration procedure. This determines whether the data are sufficiently reliable to be included in the analysis.

To estimate the accuracy in eye-movement-position data, we processed the starting and ending offsets of a subject performing 25 successive experimental trials during a $30-$ min period of reading recordings over $12^{\circ}$ of visual angle.
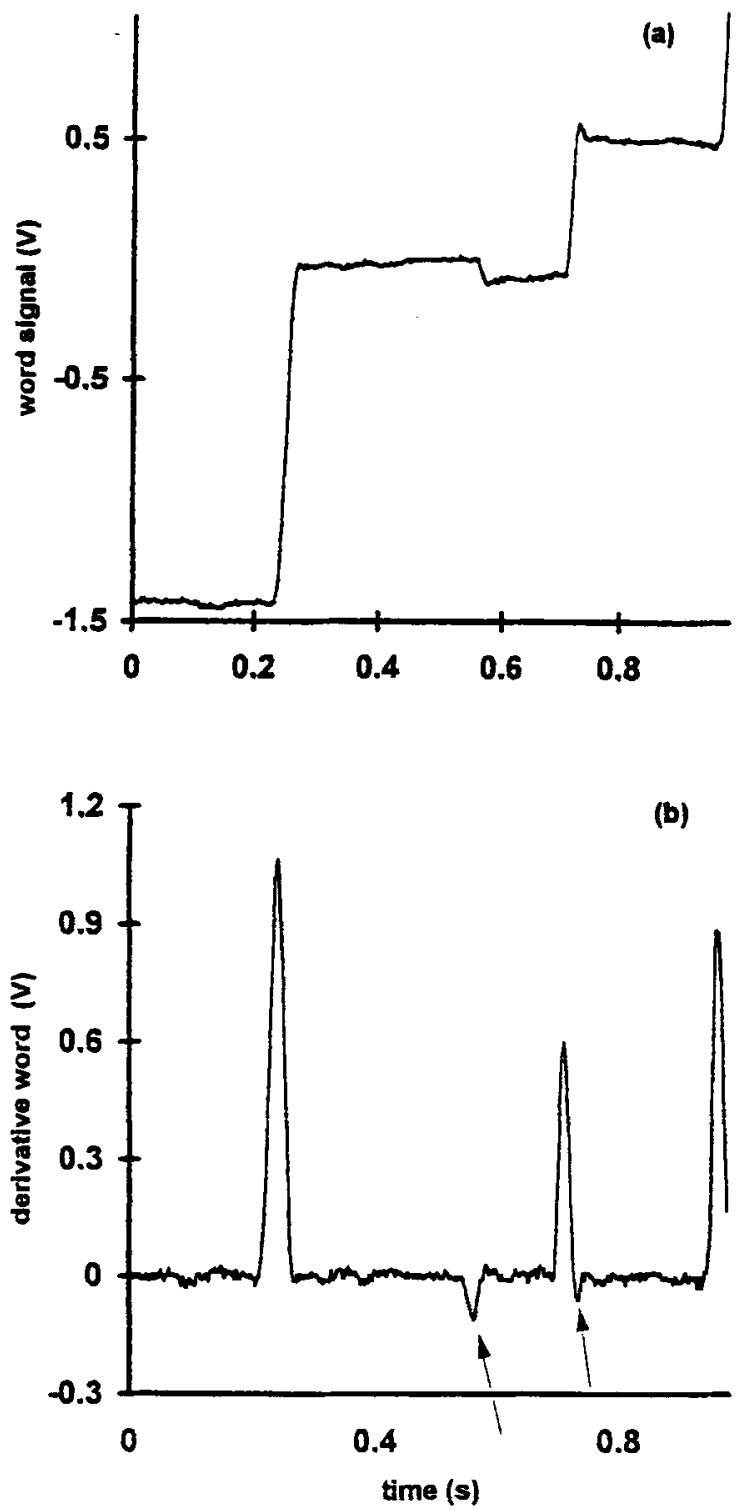

Figure 8. Eye-movement signal (top) and derivative (bottom) over time during the reading of a seven-letter word.

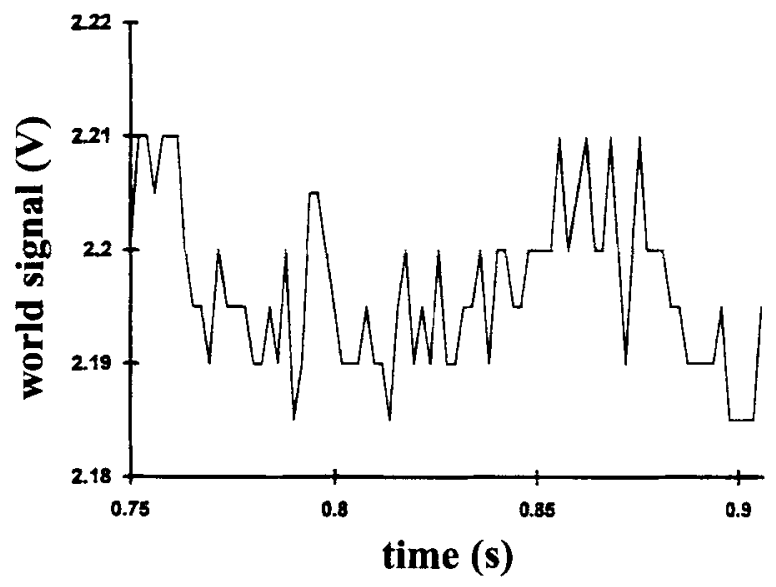

Figure 9. Noise in the eye signal over time during the last fixation on the seven-letter word shown in Figure 8.

Figure 10 presents starting- and ending-offset drifts around their mean voltage values for the 25 successive recordings. The $S D$ of the starting offset is $24 \mathrm{mV}$, while that of the ending offset is $79 \mathrm{mV}$. Clearly, the $S D$ over the $12^{\circ}$ experimental visual angle is due to the $S D$ of the ending offset. To reduce the contribution of this error to the estimation of the eye position during reading, we determined precisely the eye positions with the starting offset of each experimental trial and the calibration factor of the last calibration task. This $S D$ of the starting offset is mainly a result of slight long-term eye displacement which induces a small deviation in the absolute position and in the calibration factor. To determine an absoluteposition resolution, we have to take into account the $S D$ of the starting offset $(24 \mathrm{mV})$ and the 7-mV $S D$ of the fixation itself. The errors due to the fluctuation of calibration are included in the $24-\mathrm{mV} S D$, as explained earlier. The $S D$ of the absolute position, $31 \mathrm{mV}$, then corresponds to about 6 min of arc-that is, about $15 \%$ of a character space of $45 \mathrm{~min}$ of visual angle.

\section{CONCLUSION}

The method described in this paper allows precise determination of the position of the eye in visual tasks such as reading, making a saccade to single visual targets, or exploring pictures. It is possible to inject highly specific manipulations into visual targets to measure precisely the effects on the position of the eye. Eye-movement records provide an unobtrusive measure of behavior, but much research is needed to specify the relation between the eye movements and the perceptual and cognitive processes. The fact that specific aspects of the saccade programming in words have been affected by the linguistic properties of words (Beauvillain \& Doré, in press) provides some support for the necessity of using a precise measure of the position of the eye. Much work still needs to be done to discover precisely which properties of words determine fixation locations. 


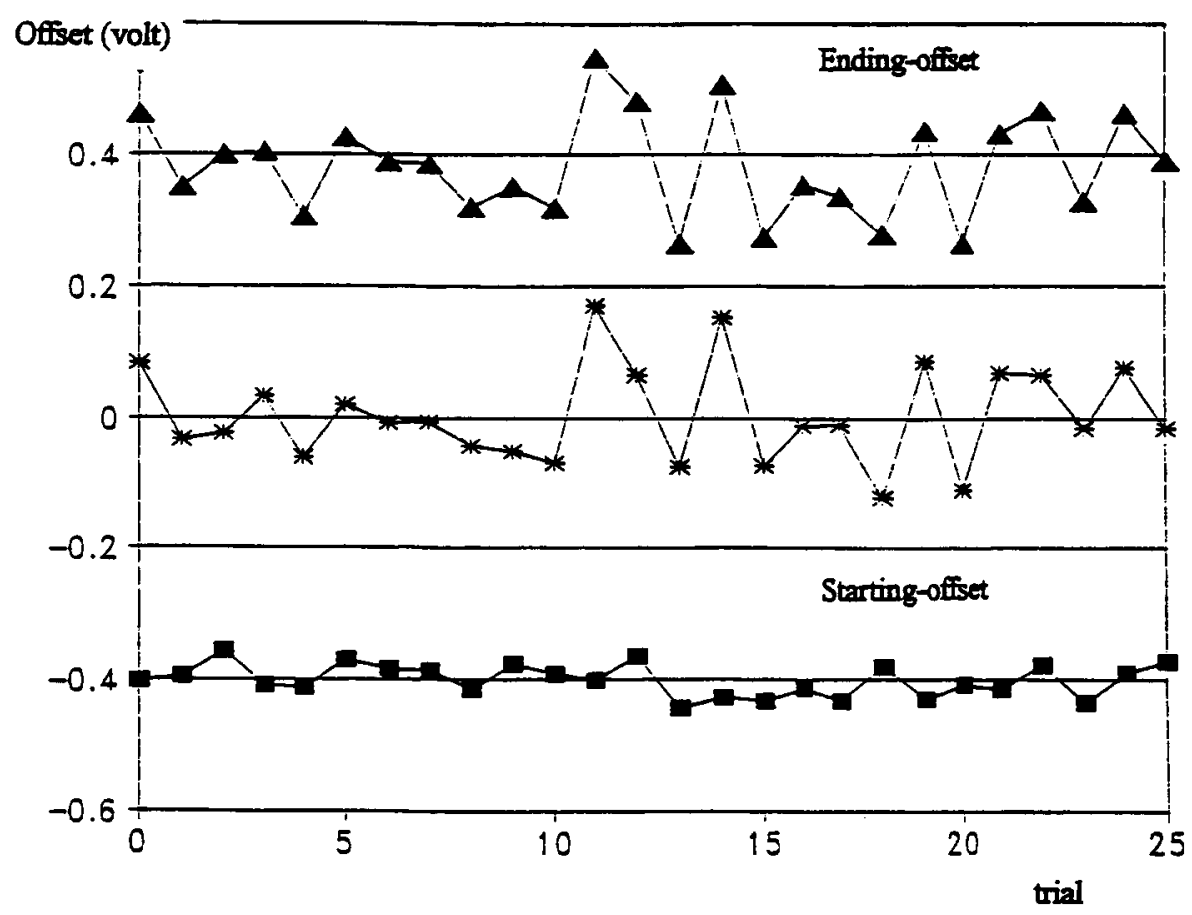

Figure 10. Mean and starting and ending offsets and difference between starting and ending offsets of a subject performing 25 successive trials during a reading experiment.

\section{REFERENCES}

BACH, M., Bouis, D., \& Fischer, B. (1983). An accurate and linear infrared oculometer. Journal of Neuroscience Methods, 9, 9-14.

Beauvillain, C., \& Doré, K. (in press). Effect of luminance and linguistic information on the center of gravity of words. In J. M. Findlay, R. W. Kentridge, \& R. Walker (Eds.), Eye movement research: Mechanisms, processes and applications. Amsterdam: NorthHolland.

Cö̈ffé, C., Humbert, R., Jacobs, A. M., \& O'Regan, J. K. (1982). Mesure des mouvements oculaires en temps réel. Informatique et Sciences Humaines, 54, 57-71.

Fischer, B., \& KunZ, B. (1981). Patterns of visually guided eye movements of trained rhesus macaques. In A. F. Fuchs \& W. Becker (Eds.), Progress in oculomotor research (pp. 49-52). Amsterdam: North-Holland.
Kapoula, Z. A., Robinson, D. A., \& Hain, T. C. (1986). Motion of the eye immediately after a saccade. Experimental Brain Research, 61, 386-394.

MCCONKIE G. W. (1981). Evaluating and reporting data quality in eye movement research. Behavior Research Methods \& Instrumentation, 13, 97-106.

Morris, R. K., Rayner, K., \& Pollatsek, A. (1990). Eye movement guidance in reading: The role of parafoveal letter and space information. Journal of Experimental Psychology: Human Perception \& Performance, 16, 268-281.

Young, L. R., \& SHEENA, D. (1975). Survey of eye movement recording methods. Behavior Research Methods \& Instrumentation, 7, $397-429$.

(Manuscript received July 21, 1993; revision accepted for publication July 28,1994 .) 